\title{
Optimization of distribution network configuration with multi objective function based on improved cuckoo search algorithm
}

\author{
Thuan Thanh Nguyen \\ Faculty of Electrical Engineering Technology, Industrial University of Ho Chi Minh City, Vietnam
}

\begin{tabular}{l} 
Article Info \\
\hline Article history: \\
Received Nov 2, 2019 \\
Revised Feb 6, 2020 \\
Accepted Apr 1, 2020 \\
\hline
\end{tabular}

\section{Keywords:}

Electric distribution network Improved cuckoo search Multi-objective Network reconfiguration Power loss reduction

\begin{abstract}
This paper proposes an improved cuckoo search (ICSA) for solving the distribution network reconfiguration (NR) problem with multi-objective function. The membership functions are considered consisting of minimizing of power loss, load balancing among branches and among the feeders, node voltage deviation and switching operation numbers. ICSA is developed from the original CSA with adding the local search mechanism for exploiting around the current best solution. The effectiveness of the ICSA has validated on the 70-node and the 83-node practical systems. The obtained results have been compared to those from runner root algorithm (RRA) and other methods in the literature. The obtained results demonstrate that ICSA has high ability for searching the optimal solution with higher successful rate and better quality of obtained solution as well as smaller iterations compared to RRA and other methods. Therefore, ICSA is a reliable method for the multi-objective NR problems.
\end{abstract}

This is an open access article under the $\underline{C C B Y-S A}$ license.

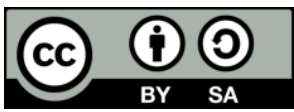

\section{Corresponding Author:}

Thuan Thanh Nguyen,

Faculty of Electrical Engineering Technology,

Industrial University of Ho Chi Minh City,

No. 12 Nguyen Van Bao, Ward 4, Go Vap District, Ho Chi Minh City, Vietnam.

Email: nguyenthanhthuan@iuh.edu.vn

\section{INTRODUCTION}

Transferring a part of loads from the heavy branches to the light branches is not only reduction of power loss but also gains a lot of advantages such as improvement of voltage profile, balance of load among branches and the feeders and enhancing the reliability of the electric distribution systems (EDS), etc. Transferring loads among branches in the EDS is implemented by changing the status of switches located in each branch. This processes is called the network reconfiguration (NR) problem. The NR problem is a nonlinear problem with constraints and a lot of local extremes. Therefore, studying the methods for solving the NR problem have attracted many concerns of the researches in more than for decades. From the first proposed by Merlin and Back [1], the NR problem has been solved by a lot of methods with different objectives.

In [2-4], the methods based on the heuristic search techniques have proposed for the NR problem with multi objective functions such as reduction of power losses, enhancement of the node voltage and increase of reliability index [2], reduction of power loss and the maximum current of the EDS [3] and optimizing the bus voltage deviations, the branch current loading, the phase current deviation and power loss of the EDS [4]. In comparisons with the heuristic methods, the number of methods based on the meta-heuristic techniques is much greater than due to a lot of advantages such as easy to implement and apply for different kind of the objective functions and the better obtained results. Therefore, more and more the meta-heuristic method are proposed for the multi objective NR problem. In [5], discrete 
particles swarm optimization (PSO) algorithm has proposed for finding the optimal configuration of the 33 and 69 nodes systems to satisfy the objective functions consisting of minimization of power loss, the number of switching operations and deviations of bus voltages. In [6], PSO is applied for the multi-objective functions consisting of reliability indexes and reduction of power loss. In [7], Binary PSO is used for finding the optimal configuration to decrease power loss and the voltage stability index. Harmony search algorithm is proposed for the NR problem for minimizing of real power losses and improvement of node voltage. In [8], adaptive genetic algorithm is used for determining the best configuration of the 69 and 136 nodes systems with the objective functions of reduction of power loss, node voltage deviation, branch current loading index, the switching numbers. In [9], the multi objective function consisting of minimizing the power losses and the reliability indices is solved by the genetic algorithm (GA). In [10], GA is also is used to solve the NR problem for reducing power loss and costs of maintenance and operation of switches. Micro GA [11] is also successful applied for finding the optimal configuration to minimize power losses and enhance a reliability index of the EDS. In [12], honey bee mating optimization is presented to reduce power loss, the switching numbers and the voltage deviation for the 33 and 86 bus systems. In [13], The objective functions such as system average interruption frequency index, average energy not supplied and active power losses are optimized by bat algorithm. In [14], grey wolf optimizer algorithm is proposed for the combined problem of network reconfiguration and STATCOM placement to reduce power loss and load balance index. The shuffled frog-leaping [15] is used for the problem of network reconfiguration with reactive power compensators. In [16], evolutionary programming, firefly, PSO and gravitational search have been successful applied for the multi objective reconfiguration to reduce power loss, enhance voltage profile and maximize the distribution generator output. In [17], the network reconfiguration considering reactive power dispatches of distributed generations (DG) is solved by non-dominated sorting PSO to reduce energy wastage from DG, power loss and voltage deviation. Reduction power loss, maximum voltage deviation, load balancing index and switching numbers of the 33 and 84 nodes system are considered by invasive weed optimization [18]. Reduction of the power loss and maintain of a continuous power supply to the customers are also considered as the multi objective function of the NR problem an solved by modified ant colony algorithms [19]. From above studies, it can be seen that with the ability for providing a good solution to NR problem, more and more recent meta-heuristic algorithms will be applied for the NR problem.

The cuckoo search algorithm (CSA) is a recent meta-heuristic inspired from the cuckoo birds' reproduction [20]. From the first proposed in 2009, CSA have been applied for a lot of problems related to the power system such as the hydrothermal scheduling problem [21], the problem of heat and power economic dispatch [22], the problem of placement of distributed generators and capacitors in the EDS $[23,24]$. For solving the NR problem, CSA has been successful applied for the problem to reduce power loss and improve voltage profile [25]. In this study, CSA outperforms PSO. In [26], CSA has been applied for the NR problem considering placement of DG. In addition, to enhance the efficient of CSA for the NR problem, the improved CSA (ICSA) has been proposed for solving the NR problem to minimize the power loss [27]. In this study, ICSA outperforms to CSA as well as many improved versions of CSA. However, the efficient of ICSA for the NR problem with other objective functions is still a considerable question.

This paper presents an NR method for multi-objective based on ICSA. The objective functions considered are reduction of power loss, enhancing of load balancing among the branches and among the feeders, node voltage deviation and the number of switching operations. The effectiveness of the ICSA for the multi-objective NR problem is evaluated on the 70-node and the practical 83-node systems. The highlighting features of the work can be summarized as follows: (1) The ICSA is proposed for the multi-objective NR problem with five above membership objective functions; (2) The selection of the final solution based on max-min method is used to compromise the membership objective functions; (3) The performance of the ICSA is effectively evaluated on the complex EDS consisting of the 70-node and the practical 83-node systems.

\section{THE PROBLEM FORMULATION OF THE MULTI OBJECTIVE NR}

There are five membership functions are considered in the paper consisting of minimizing power loss, improving load balancing among the branches, load balancing among the feeders, improving node voltages and reducing the number of switching operations. Each membership function is determined as follows:

\subsection{Reduction of power loss $(\Delta \mathrm{P})$}

Power loss reduction is the most part of the NR problem which is determined as follows: 


$$
\Delta \mathrm{P}=\sum_{i=1}^{N b r} k_{i} \cdot R_{i} \cdot\left(\frac{P_{i}^{2}+Q_{i}^{2}}{V_{i}^{2}}\right)
$$

Where $N_{b r}$ is the branch number of the EDS. $R_{i}, V_{i}, P_{i}$ and $Q_{i}$ are the resistance, the ending voltage, the active and reactive power flow of the $i$ th branch, respectively.

\subsection{Balance of loads among the branches (LBI)}

Balance of loads among the branches helps the load carrying level of each branch in the EDS is approximately equal. Due to the different rate current of each branch, to balance the load carrying level of each branch, LBI is determined as follows [18, 28]:

$$
L B I=\operatorname{var}\left[\frac{I_{1}}{I_{1, \max }}, \frac{I_{2}}{I_{2, \max }}, \ldots \frac{I_{i}}{I_{i, \max }}, \ldots, \frac{I_{n}}{I_{n, \max }}\right]
$$

Where var called variance, $I_{i}$ and $I_{i, \max }$ are the current and the rate current of the branch $i$ th, respectively.

\subsection{Balance of loads among the feeders (LBF)}

LBF is considered to support the EDS transfer loads from the heavy feeders to light feeders, it is determined as follows:

$$
L B F=\operatorname{var}\left[I_{F, 1}, I_{F, 2}, \ldots I_{F, i}, \ldots, I_{F, N F}\right]
$$

Where $I_{F, i}$ is a current of the $i$ th feeder, $N F$ is the total number of feeders.

\subsection{Node voltage deviation}

To enhance power quality of the system, the node voltage deviation $(\Delta \mathrm{V})$ is also considered to an objective of the NR problem. $\Delta \mathrm{V}$ is calculated as follows:

$$
\Delta V=V_{\text {ref }}-V_{\min }
$$

Where $V_{\text {ref }}$ and $V_{\min }$ are the feeders voltage and the minimum node voltage in per unit of the EDS

\subsection{Number of switching operations (NSW)}

For decreasing the operating cost, NSW is also considered as one of the objective function. Which is determined as follows [18]:

$$
N S W=\sum_{i=1}^{N b r}\left|S_{0, i}-S_{i}\right|
$$

Where, $S_{0, i}$ and $S_{i}$ are status of the switch $i$ th before and after performing reconfiguration.

\subsection{The objective function}

The final compromise solution (FCS) for five membership functions (MF) is determined by the max-min method $[18,29,30]$ as follows:

$$
M F_{k}(X)= \begin{cases}1, & F_{k}(X) \leq F_{k}^{\text {min }} \\ \frac{F_{k}^{\text {max }}-F_{k}(X)}{F_{k}^{\text {max }}-F_{k}^{\text {min }},}, & F_{k}^{\text {min }}<F_{k}(X)<F_{k}^{\text {max }} \\ 0, & F_{k}(X) \geq F_{k}^{\text {max }}\end{cases}
$$

In which, the maximum value of the membership functions $F_{k}^{\max }$ are calculated from the initial configuration because the purposed of the NR problem is finding the configuration having the better objective function value than the initial configuration. The $F_{k}^{\min }$ of the membership functions are determined by solving the NR problem with single objective function.

The degree of overall satisfaction for each candidate solution is the minimum of all of the membership functions. Then, due to the membership functions value of less than unity, the fuzzy decision for the candidate configuration is as follows:

$$
F C S(X)=1-\min \left\{M F_{k}(X)\right\}
$$


Furthermore, each candidate configuration must be satisfied the following constraints: The radial topology of the EDS must be maintained and all of nodes must be served and all of nodes' voltage must be in permissible ranges as well as no branch is over load.

\section{ICSA FOR THE MULTI OBJECTIVE NR PROBLEM}

The application of ICSA for the multi-objective NR is described in more details as follows:

Step 1: Initialization

At the beginning, the population of network configuration is generated as follows:

$$
X_{i}=\mathrm{fr}\left[X_{l o, j}+r_{1} \cdot\left(X_{h i, j}-X_{l o, j}\right)\right]
$$

Where $\mathrm{X}_{\mathrm{i}}$ is the candidate configuration $i$ th with $\mathrm{i}=1,2, \ldots, \mathrm{N} . \mathrm{N}$ is population size. $f r$ is a round function. $r_{l}$ is a random number in $[0,1] . X_{h i, j}$ and $X_{l o, j}$ are the upper and lower bounds of the switch $j$ th with $j=1,2, \ldots$, $D . D$ is the number of opened switches in the EDS.

From the created population, the fitness function shown in (7) is evaluated and the current best solution $\mathrm{X}_{\text {gbest }}$ is determined.

\section{Step 2: Creation of new population based on Lévy flight}

In this step, a new population network configuration is created by the Lévy flight as follows:

$$
X_{i}^{\text {new }}=\mathrm{fr}\left[\mathrm{Xbest}_{i}+r_{2} \times \Delta X_{i}^{\text {new }}\right]
$$

Where, $\mathrm{R}_{2}$ is a random number in $[0,1] . \Delta X_{i}^{\text {new }}$ is an increased value determined by the Lévy movement [20]. Xbest $_{i}$ is the so far best solution of the configuration $i$ th in the population.

Similar to step 1, the fitness function is evaluated. Then the current population is updated if the new individual is better than the corresponding once in the current population and the current best solution $X_{\text {gbest }}$ is updated.

\section{Step 3: Creation of new population based on alien eggs discovery}

In this step, there is also a new population generated as follows:

$$
X_{i}^{\text {new }}=f r\left[\text { Xbest }_{i}+K(i,:) \otimes \Delta X_{i}^{\text {new }}\right]
$$

Where $\Delta X_{i}^{\text {new }}$ is an increased value determined by the random movement [20]. $\mathrm{K}$ is an matrix with the value of elements of 0 or 1 controlled by the $\mathrm{P}_{\mathrm{a}}>0$ factor:

$$
K=\operatorname{rand}(N, D)>P_{a}
$$

From the new population, the fitness function is evaluated. Then the current population is updated if the new individual is better than the corresponding once in the current population and the current best solution $X_{\text {gbest }}$ is updated.

\section{Step 4: Creation of new solutions around the current best solution}

In this step, there are D new solutions is created by modified one by one variable of the current best solution $X_{\text {gbest }}$. In which, the value of the modified control variable is controlled by the distance $\mathrm{d}$ from the current to new variable [27]. In ICSA, the value of $\mathrm{d}$ is set to the $d_{\max }$ and $d_{\min }$, so there are 2.D new solutions are created around the $X_{\text {gbest }}$.

$$
X_{n e w, j}=\operatorname{fr}\left[K_{(j,:)} \cdot d \cdot \mathrm{r}_{3}+X_{g b e s t}(i)\right] \text { with } j=1,2, \ldots, D
$$

Where $K_{(j,:)}$ is a vector with all elements of 0 except the element $j$ th set to $1 . \mathrm{R}_{3}$ is a random number in $[0,1]$.

In addition, to exploit more the search space around the $X_{g b e s t}$, the $K_{(j,:)}$ is also replaced by the random vector $K r_{(j,:)}$ which has more than one element set to 1 . Therefore, there are also 2.D new solutions are created around the $\mathrm{X}_{\mathrm{gbest}}$ by modified more than one control variable of the $\mathrm{X}_{\text {gbest. }}$. From the 4.D new solutions, the fitness function is evaluated and the current best solution $X_{g b e s t}$ is updated.

\section{Step 5: Stopping the ICSA for the NR problem}

The searching processes of new configuration will be stopped as the number of evaluation the fitness function is reached to the pre-set value $(M F E)$. In addition, because of taking long time for calculating the fitness function, the number of iterations called (Iter not-improved $_{\text {) }}$ that the fitness function value is not be improved is also considered as another stopping condition for ICSA. If two above conditions are not satisfied the ICSA will move to step 2 for searching elsewhere ICSA will be stopped and the best configuration $X_{\text {gbest }}$ is reported. 


\section{RESULTS AND DISCUSSION}

In this section, there are two illustrative system is used to reconfigure. The first system is used to validate the effectiveness of ICSA while the second system which is a practical system is used to show the application potential of ICSA for the practical systems.

\subsection{The 70-node system}

To evaluate effectiveness of ICSA for the multi-objective NR problem, the 70-node system consisting of 4 feeders, 78 branches and 11 opened switches shown in Figure 1 is used to reconfigure. The data of the system are referenced from [31]. In the initial configuration with opened switches of $\{69,70$, $71,72,73,74,75,76,77,78,79\}$, the power loss of the system is $227.53 \mathrm{~kW}$. The proposed ICSA method is implemented in Matlab software on the personal computer Intel(R) Core(TM) i5-2430 CPU@2.4GHz with 4GB RAM. In addition, the runner root algorithm (RRA) is also run on the same computer to compare with ICSA. Due to successful application of RRA for the multi objective NR problem [32], RRA is selected for comparison. For ICSA, the $P_{a}$ in (11), the $d_{\max }$ and $d_{\min }$ in (12) are set to 0.2, 4 and 2 respectively. For RRA, all parameters are selected similar to [32]. Some parameters selected the same for both ICSA and RRA are that the population size $N=30$, the stopping condition $M F E=30000$ and Iter $_{\text {not-improved }}=200$.

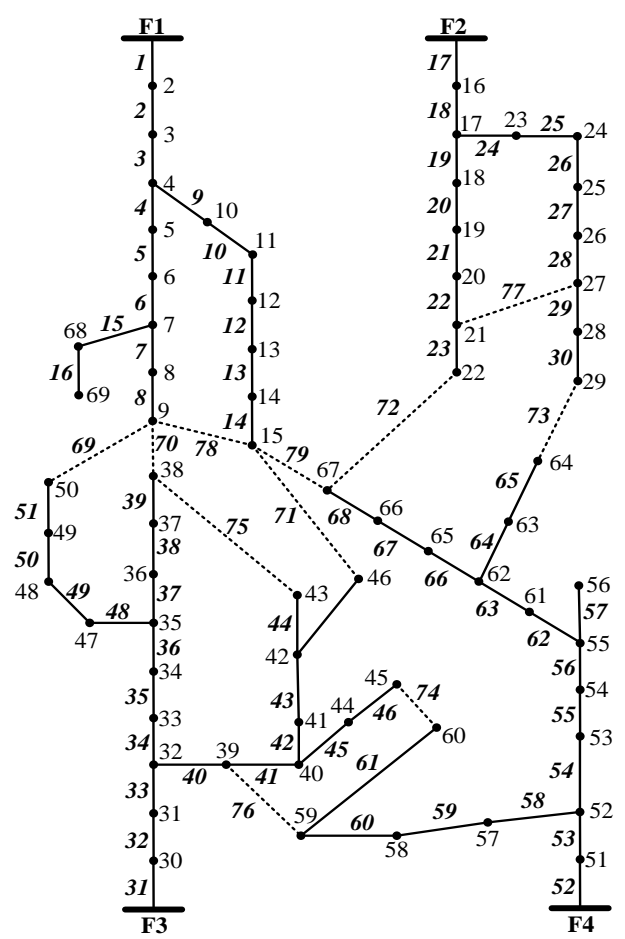

Figure 1.70-node test system

The performance of the ICSA is presented in Table 1. By using ICSA, the opened switches obtained in the optimal configuration are $\{46,51,67,70,71,73,75,76,77,78$, and 79$\}$. After reconfiguration, the power loss has been reduced from 227.5625 to $205.6133 \mathrm{~kW}$, the minimum voltage has also been improved from 0.9052 to 0.9283 p.u. Furthermore, LBI is reduced from 0.0083 to 0.0069 and the maximum load carrying coefficient is decreased from 0.3471 to 0.2890 . Specially, to achieve the aforementioned results, it only takes 6 changing switches. The voltage and current profiles illustrated in Figure 2 shows that most of node voltages have been enhanced and the load balance among branches is more improved after performing reconfiguration by ICSA.

Regarding the effectiveness of ICSA for multi-objective NR problems, Table 1 shows that although both ICSA and RRA have determined the optimal configuration, it is clear that the effectiveness of ICSA is superior to RRA. Specifically, in 50 independent runs, ICSA has found the optimal configuration in 24 runs, while the RRA has reached the optimal solution only in 3 runs. These result leads to poor quality of obtained solution from RRA compared to ICSA. The maximum, mean and standard deviations (STD) of the adaptive function obtained from ICSA are much smaller than those of RRA. They are 0.5261, 0.2857

Optimization of distribution network configuration with multi objective function... (Thuan Thanh Nguyen) 
and 0.0427 respectively for ICSA while their values for RRA are $0.6951,0.3870$ and 0.1089 . In addition, the average number of converged iterations of ICSA is also less than that of RRA. The maximum, minimum and mean convergence characteristics of both methods in 50 runs are shown in Figure 3 . The figure demonstrates that the corresponding convergence curves of ICSA converge to smaller values than RRA. In addition, the mean convergence curve of ICSA is much closed to the minimum convergence curve. This shows the stability of ICSA for the multi-objective NR problem.

Table 1. The comparisons among ICSA with RRA for the 70-node system

\begin{tabular}{llll}
\hline \multicolumn{1}{c}{ Item } & \multicolumn{1}{c}{ Initial } & \multicolumn{1}{c}{ ICSA } & \multicolumn{1}{c}{ RRA } \\
\hline Optimal configuration & $69,70,71,72,73,74$, & $46,51,67,70,71,73$, & $46,51,67,70,71,73$, \\
$75,76,77,78,79$ & 205.6133 \\
Ploss (kW) & $75,76,77,78,79$ & $75,76,77,78,79$ & $0.9283(29)$ \\
V $_{\text {min }}(\mathrm{pu})$ (node) & 227.5625 & 205.6133 & 0.0069 \\
LBI & 0.9052 & $0.9283(29)$ & 0.2890 \\
Max (I/Irate) & 0.0083 & 0.0069 & 0.0086 \\
LBF & 0.3471 & 0.2890 & {$[1.2863,1.4449,1.4735$,} \\
LBFi (MW) & 0.1107 & 0.0086 & $1.4865]$ \\
NSW & {$[1.0991,1.1992,1.7853$,} & {$[1.2863,1.4449,1.4735$,} & 6 \\
Number of runs found out the optimal & - & $1.4865]$ & $3 / 50$ \\
solution/total runs & 0 & 6 & 0.6951 \\
Max of fitness & - & $24 / 50$ & 0.2727 \\
Min of fitness & 1 & 0.5261 & 0.3870 \\
Mean of fitness & - & 0.2727 & 0.1089 \\
STD of fitness & - & 0.2857 & 118 \\
The number of convergence iterations & - & 0.0427 & 97.7875 \\
CPU times (second) & - & 60 & 162.0978 \\
\hline
\end{tabular}

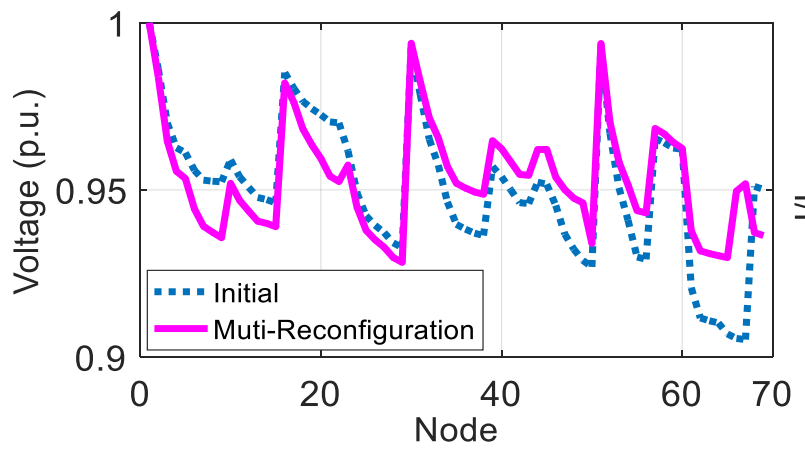

(a)

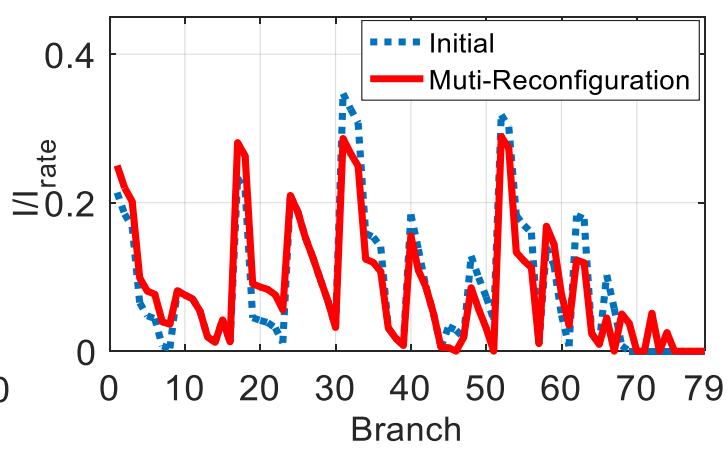

(b)

Figure 2. Voltage and current profile of the 70-node system before and after reconfiguration; (a) voltage profile, (b) current profile

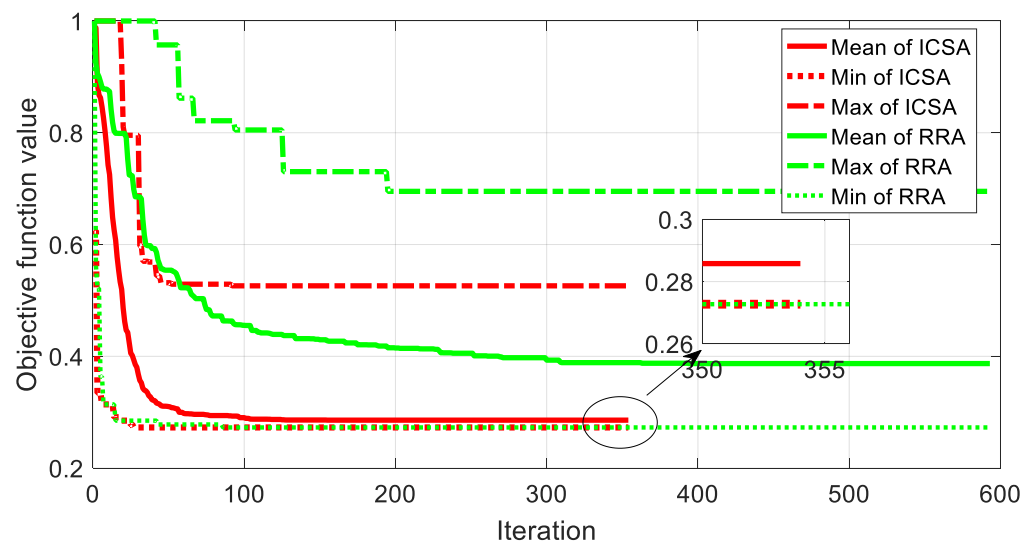

Figure 3. Convergence characters of ICSA and RRA for the 70-node system 
The comparisons among ICSA with other method in literature are shown in Table 2 also lead to the better obtained solution. Although the indexes of $V_{\min }, L B I, L B F$ and $N S W$ obtained from Multi-RRA [32] is nearly the same with ICSA, power loss obtained from Multi-RRA is still higher than that of ICSA. Compared to hybrid PSO (HPSO) [33], combination of heuristic rules and fuzzy multi objective approach (FMA) [31] and combination of honey bee mating optimization-discrete PSO (DPSO-HBMO) [34], all of indexes are similar to those of ICSA however it only takes 6 switching numbers compared to 12 of compared methods. In addition the balance of among memberships functions of ICSA compared to other methods shown in Table 3 demonstrated that the balance of among membership functions of ICSA is better than to others. Variance (var) of the membership function values obtained by ICSA is the smallest among the compared methods.

Table 2. The comparisons among ICSA with other methods

\begin{tabular}{|c|c|c|c|c|c|c|c|c|}
\hline Method & $\begin{array}{l}\text { Optimal opened } \\
\text { switches }\end{array}$ & $\begin{array}{c}\Delta \mathrm{P} \\
(\mathrm{kW})\end{array}$ & $\begin{array}{l}\mathrm{V}_{\min } \\
\text { (p.u.) }\end{array}$ & LBI & $\begin{array}{c}\text { Max } \\
\text { (I/Irate) }\end{array}$ & $\mathrm{LBF}$ & $\mathrm{LBF}_{\mathrm{i}}$ & NSW \\
\hline ICSA & $\begin{array}{c}46,51,67,70,71,73 \\
75,76,77,78,79\end{array}$ & 205.61 & 0.9283 & 0.0069 & 0.2890 & 0.0086 & $\begin{array}{c}{[1.2863,1.4449,} \\
1.4735,1.4865]\end{array}$ & 6 \\
\hline HPSO [33] & $\begin{array}{c}14,79,71,39,51,28 \\
73,67,46,76,70\end{array}$ & 205.07 & 0.9273 & 0.0066 & 0.2890 & 0.0085 & $\begin{array}{c}{[1.2871,1.4471,} \\
1.4733,1.4865]\end{array}$ & 12 \\
\hline $\begin{array}{c}\text { DPSO-HBMO } \\
{[34]}\end{array}$ & $\begin{array}{c}14,79,71,39,51,28 \\
73,67,46,76,70\end{array}$ & 205.07 & 0.9273 & 0.0066 & 0.2890 & 0.0085 & $\begin{array}{c}{[1.2871,1.4471,} \\
1.4733,1.4865]\end{array}$ & 12 \\
\hline
\end{tabular}

Table 3. Balance among memberships functions of ICSA compared to other methods

\begin{tabular}{ccccccc}
\hline Method & $\mathrm{MF}_{\text {Ploss }}$ & $\mathrm{MF}_{(1-\mathrm{V} \min )}$ & $\mathrm{MF}_{\mathrm{LBI}}$ & $\mathrm{MF}_{\mathrm{NSW}}$ & $\mathrm{MF}_{\mathrm{LBF}}$ & $\operatorname{var}(\mathrm{MF})$ \\
\hline ICSA & 0.83911 & 0.83863 & 0.73684 & 0.72727 & 0.92455 & 0.0067 \\
Multi-RRA [32] & 0.7359 & 0.8384 & 0.7182 & 0.72727 & 0.9139 & 0.0074 \\
HPSO [33] & 0.8601 & 0.8016 & 0.6499 & 0.4545 & 0.9252 & 0.0355 \\
FMA [31] & 0.8601 & 0.8016 & 0.6499 & 0.4545 & 0.9252 & 0.0355 \\
DPSO-HBMO [34] & 0.8601 & 0.8016 & 0.6499 & 0.4545 & 0.9252 & 0.0355 \\
\hline
\end{tabular}

\subsection{The 83-node practical system}

The second system which is a practical system of the Taiwan Power Company. There are 83 nodes, 11 feeders and 13 open switches. The single line diagram and data of the system is referenced from [35]. At the initial radial configuration, power loss is $531.9924 \mathrm{~kW}$ and minimum voltage is 0.9285 p.u. Because of lack of rate current, the LBI membership is ignored. For ICSA, the population size and the stopping condition is chosen to 40 and 40000, respectively. Other control parameters of ICSA are selected similar to those of the 70-node system.

The performance of the ICSA is presented in Table 4. By using ICSA, the opened switches obtained in the optimal configuration are $\{7,34,63,84,86,87,88,89,90,91,92,93$, and 95$\}$. After reconfiguration, the power loss has been reduced from 531.9924 to $477.0592 \mathrm{~kW}$, the minimum voltage has also been improved from 0.9285 to 0.9479 p.u. Furthermore, LBF is reduced from 1.4418 to 0.7124 . The maximum power difference among feeders has decreased from 3.2840 to 2.7044 MVA. Specially, to achieve the aforementioned results, it only takes 6 changing switches. The voltage profiles illustrated in Figure 4 show that most of node voltages have been enhanced after performing reconfiguration by ICSA.

Table 4. The application of ICSA for the practical 83-node system

\begin{tabular}{ccc}
\hline Item & Initial & ICSA \\
\hline Open switches & $84,85,86,87,88,89,90,91,92,93,94,95,96$ & $7,34,63,84,86,87,88,89,90,91,92,93,95$ \\
Ploss (kW) & 531.9924 & 477.0592 \\
$\mathrm{~V}_{\text {min }}(\mathrm{pu})(\mathrm{node})$ & $0.9285(10)$ & $0.9479(84)$ \\
LBF & 1.4418 & 0.7124 \\
LBFi (MW) & {$[4.4317,3.3764,4.5232,2.8043,4.6394,1.3554$,} & {$[2.5865,3.3764,4.5232,2.8043,2.9195,3.0325$} \\
NSW & $3.3428,1.8486,3.5155,1.9114,4.6158]$ & $4.1470,2.8228,3.5155,1.9114,4.6158]$ \\
\end{tabular}

Optimization of distribution network configuration with multi objective function... (Thuan Thanh Nguyen) 


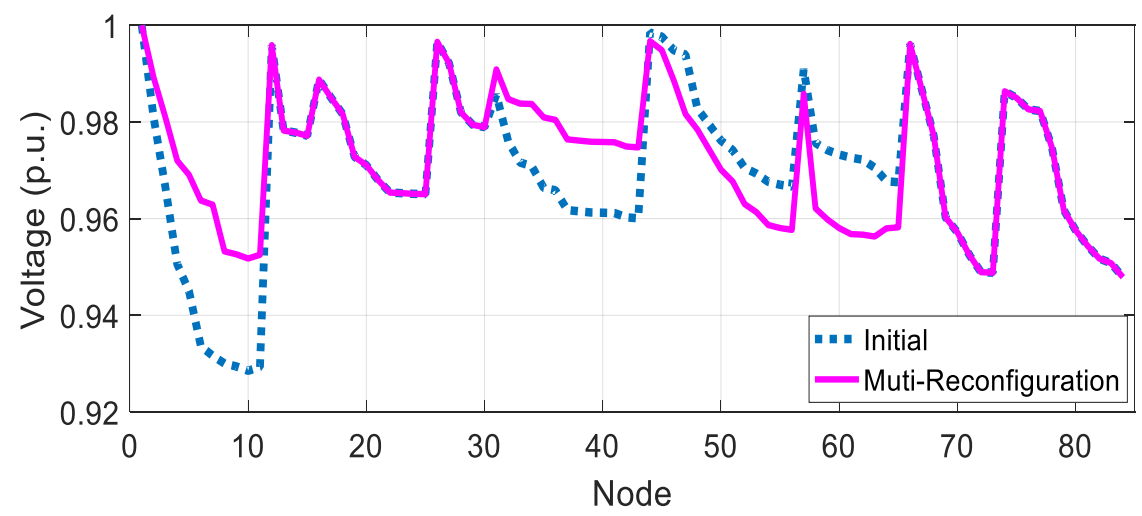

Figure 4. Voltage profile of the 83-node practical system

\section{CONCLUSION}

In this paper, the application of ICSA for the multi-objective NR problem has been successfully implemented for minimizing power loss, load balancing among the branches and among the feeders, node voltage deviation and switching operation numbers. The max-min method is used to selecte best compromise solution. The proposed method has been evaluated on the complex 70-node system. The numerical results validate that ICSA outperforms to RRA in terms of success rate, the obtained optimal solution quality and the number of converged iterations and other methods in literature in term of the quality of membership function values. In addition, ICSA is also applied for the practical system and the obtained results is more effective than the initial configuration. Therefore, ICSA can be the efficient and promised for solving the NR problems.

\section{REFERENCES}

[1] A. Merlin, "Search for a minimal loss operating spanning tree configuration in an urban power distribution system," Proceeding in 5th power system computation conf (PSCC), Cambridge, UK, vol. 1, pp. 1-18, 1975.

[2] D. P. Bernardon, V. J. Garcia, A. S. Q. Ferreira, and L. N. Canha, "Electric distribution network reconfiguration based on a fuzzy multi-criteria decision making algorithm," Electric Power Systems Research, vol. 79, no. 10, pp. 1400-1407, October 2009

[3] L. S. M. Guedes, A. C. Lisboa, D. A. G. Vieira and R. R. Saldanha, "A Multiobjective Heuristic for Reconfiguration of the Electrical Radial Network," in IEEE Transactions on Power Delivery, vol. 28, no. 1, pp. 311-319, Jan. 2013.

[4] G. Mahendran, M. Sathiskumar, S. Thiruvenkadam, and L. Lakshminarasimman, "Multi-objective Unbalanced Distribution Network Reconfiguration through Hybrid Heuristic Algorithm," Journal of Electrical Engineering and Technology, vol. 8, no. 2, pp. 215-222, 2013.

[5] M. R. Andervazh, J. Olamaei, M. R. Haghifam, “Adaptive multi-objective distribution network reconfiguration using multi-objective discrete particles swarm optimisation algorithm and graph theory," IET Generation, Transmission \& Distribution, vol. 7, no. 12, pp. 1367-1382, December 2013.

[6] Amir Sabbagh Alvani, Seyed Mehdi Mahaei, "Reconfiguration of distribution networks with presence of DGs to improving the reliability," Bulletin of Electrical Engineering and Informatics, vol. 5, no. 1, pp. 17-24, March 2016.

[7] Aji Akbar Firdaus, Ontoseno Penangsang, Adi Soeprijanto, Dimas Fajar U.P., "Distribution network reconfiguration using binary particle swarm optimization to minimize losses and decrease voltage stability index," Bulletin of Electrical Engineering and Informatics, vol. 7, no. 4, pp. 514-521, December 2018.

[8] N. Gupta, A. Swarnkar, K. R. Niazi, R. C. Bansal, "Multi-objective reconfiguration of distribution systems using adaptive genetic algorithm in fuzzy framework," IET Generation, Transmission \& Distribution, vol. 4, no. 12, pp. 1288-1298, 2010.

[9] N. G. Paterakis et al., "Multi-Objective Reconfiguration of Radial Distribution Systems Using Reliability Indices," in IEEE Transactions on Power Systems, vol. 31, no. 2, pp. 1048-1062, March 2016.

[10] H. Souifi, O. Kahouli, and H. Hadj Abdallah, "Multi-objective distribution network reconfiguration optimization problem," Electrical Engineering, vol. 101, pp. 45-55, 2019.

[11] J. Mendoza, M. López, and E. López, "Multiobjective Reconfiguration Considering Power Losses and Reliability Index for Distribution Network," IET Generation, Transmission \& Distribution, vol 3, no. 9, pp. 825-840, 2009.

[12] T. Niknam, "An efficient multi-objective HBMO algorithm for distribution feeder reconfiguration," Expert Systems with Applications, vol. 38, no. 3, pp. 2878-2887, 2011.

[13] A. Kavousi-Fard and T. Niknam, "Multi-objective stochastic Distribution Feeder Reconfiguration from the reliability point of view," Energy, vol. 64, pp. 342-354, 2014. 
[14] G. Selvaraj and K. Rajangam, "Multi-objective grey wolf optimizer algorithm for combination of network reconfiguration and D-STATCOM allocation in distribution system," International Transactions on Electrical Energy Systems, vol. 29, no. 11, pp. 1-21, November 2019.

[15] H. D. Dehnavi and S. Esmaeili, "A new multiobjective fuzzy shuffled frog-leaping algorithm for optimal reconfiguration of radial distribution systems in the presence of reactive power compensators," Turkish Journal of Electrical Engineering and Computer Sciences, vol. 21, no. 3, pp. 864-881, 2013.

[16] O. Badran, H. Mokhlis, S. Mekhilef, and W. Dahalan, "Multi-Objective Network Reconfiguration with Optimal DG Output Using Meta-Heuristic Search Algorithms," Arabian Journal for Science and Engineering, vol. 43, no. 6, pp. 2673-2686, 2018.

[17] W. Ongsakul, J. G. Singh, and S. R. Tuladhar, "Multi-objective approach for distribution network reconfiguration with optimal DG power factor using NSPSO," IET Generation, Transmission \& Distribution, vol. 10, no. 12, pp. 2842-2851, 2016.

[18] D. Sudha Rani, N. Subrahmanyam, and M. Sydulu, "Multi-Objective Invasive Weed Optimization - An application to optimal network reconfiguration in radial distribution systems," International Journal of Electrical Power \& Energy Systems, vol. 73, pp. 932-942, 2015.

[19] A. Oloulade, A. I. Moukengue, R. Agbokpanzo, A. Vianou, H. Tamadaho, and R. Badarou, "New Multi Objective Approach for Optimal Network Reconfiguration in Electrical Distribution Systems Using Modified Ant Colony Algorithm," American Journal of Electrical Power and Energy Systems, vol. 8, no. 5, pp. 120-126, 2019.

[20] X. Yang and Suash Deb, "Cuckoo Search via Lévy flights," 2009 World Congress on Nature \& Biologically Inspired Computing (NaBIC), Coimbatore, pp. 210-214, 2009.

[21] T. T. Nguyen, D. N. Vo, and A. V. Truong, "Cuckoo search algorithm for short-term hydrothermal scheduling," Applied Energy, vol. 132, pp. 276-287, 2014.

[22] T. T. Nguyen, D. N. Vo, and B. H. Dinh, "Cuckoo search algorithm for combined heat and power economic dispatch," International Journal of Electrical Power \& Energy Systems, vol. 81, pp. 204-214, 2016.

[23] Z. Moravej and A. Akhlaghi, "A novel approach based on cuckoo search for DG allocation in distribution network," International Journal of Electrical Power and Energy Systems, vol. 44, no. 1, pp. 672-679, 2013.

[24] O. P. Mahela, S. Ram, and O. Lalit, "Optimal Capacitor Placement for Loss Reduction in Radial Distribution Feeder," IOSR Journal of Electrical and Electronics Engineering, vol. 4, no. 6, pp. 43-48, 2013.

[25] T. T. Nguyen and A. V. Truong, "Distribution network reconfiguration for power loss minimization and voltage profile improvement using cuckoo search algorithm," International Journal of Electrical Power and Energy Systems, vol. 68, pp. 233-242, 2015.

[26] T. T. Nguyen, A. V. Truong, and T. A. Phung, "A novel method based on adaptive cuckoo search for optimal network reconfiguration and distributed generation allocation in distribution network," International Journal of Electrical Power \& Energy Systems, vol. 78, pp. 801-815, 2016.

[27] T. T. Nguyen and T. T. Nguyen, "An improved cuckoo search algorithm for the problem of electric distribution network reconfiguration," Applied Soft Computing, vol. 84, pp. 1-28, November 2019.

[28] M. Sedighizadeh, S. Ahmadi, and M. Sarvi, "An Efficient Hybrid Big Bang-Big Crunch Algorithm for Multiobjective Reconfiguration of Balanced and Unbalanced Distribution Systems in Fuzzy Framework," Electric Power Components and Systems, vol. 41, no. 1, pp. 75-99, 2013.

[29] K. Nekooei, M. M. Farsangi, H. Nezamabadi-Pour and K. Y. Lee, "An Improved Multi-Objective Harmony Search for Optimal Placement of DGs in Distribution Systems," in IEEE Transactions on Smart Grid, vol. 4, no. 1, pp. 557-567, March 2013.

[30] M. Haghifam, H. Falaghi, and O. Malik, "Risk-based distributed generation placement," Generation, Transmission \& Distribution, IET, vol. 2, no. 2, pp. 252-260, 2008.

[31] D. Das, "A fuzzy multiobjective approach for network reconfiguration of distribution systems," in IEEE Transactions on Power Delivery, vol. 21, no. 1, pp. 202-209, Jan. 2006.

[32] T. T. Nguyen, T. T. Nguyen, A. V. Truong, Q. T. Nguyen, and T. A. Phung, "Multi-objective electric distribution network reconfiguration solution using runner-root algorithm," Applied Soft Computing, vol. 52, pp. 93-108, 2017.

[33] T. Niknam, "An efficient hybrid evolutionary algorithm based on PSO and ACO for distribution feeder reconfiguration," European Transactions On Electrical Power, vol. 20, pp. 575-590, 2009.

[34] T. Niknam, "An efficient hybrid evolutionary algorithm based on PSO and HBMO algorithms for multi-objective Distribution Feeder Reconfiguration," Energy Conversion and Management, vol. 50, no. 8, pp. 2074-2082, 2009.

[35] Ching-Tzong Su and Chu-Sheng Lee, "Network reconfiguration of distribution systems using improved mixedinteger hybrid differential evolution," in IEEE Transactions on Power Delivery, vol. 18, no. 3, pp. 1022-1027, July 2003 .

\section{BIOGRAPHY OF AUTHOR}

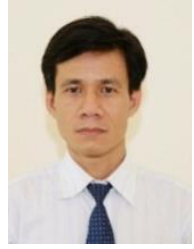

Thuan Thanh Nguyen received his M.Eng. and Ph.D degrees in electrical engineering from Ho Chi Minh City University of Technology and Education, Viet Nam in 2012 and 2018 respectively. Currently, he is a lecturer at Faculty of Electrical Engineering Technology, Industrial University of Ho Chi Minh City, Viet Nam. His research fields are power system optimization, optimization algorithms, and renewable energies. 